\title{
Theoretische Informatik
}

Ein Themenheft des Informatik-Spektrums über theoretische Informatik? Manche werden vielleicht an ein unangenehmes Prüfungsfach zurückdenken, andere meinen, da ginge es hauptsächlich um Turingmaschinen, endliche Automaten und die Chomsky-Hierarchie.

In der Tat ist es ",von außen" schwer, die Vielfalt der theoretischen Informatik und ihre Fortschritte zu verfolgen - zu schnell ist die Entwicklung.

Ein treffendes Bild der theoretischen Informatik zu vermitteln und Eindrücke von den faszinierenden aktuellen Forschungsfragen zu geben ist das Anliegen des vorliegenden Themenhefts. Es wendet sich gerade an diejenigen Leserinnen und Leser, die wenig über die komplexe Landschaft der heutigen theoretischen Informatik wissen, und folglich ist die Darstellung in den meisten Beiträgen recht informell gehalten.

- In einem Abriss Theoretische Informatik - ein Kurzprofil skizzieren wir den Zuschnitt und Tendenzen der theoretischen Informatik - trotz der grundlegenden Schwierigkeiten eines solchen Unterfangens angesichts der Vielfältigkeit der Teilgebiete und der Überschneidungen mit Nachbardisziplinen innerhalb und außerhalb der Informatik.

Es folgen Artikel, die sich ausgewählten Teilbereichen der theoretischen Informatik widmen:

- Susanne Albers berichtet über Fortschritte im Bereich der Onlinealgorithmen, also der Algorithmen, die Berechnungen bereits durchführen, während nach und nach die Eingabedaten eintreffen.

- Franz Baader, Bernhard Beckert und Tobias Nipkow beleuchten verschiedene Aspekte der Deduktion, insbesondere interaktive Beweiser, automatische Deduktion und logikbasierte Wissensrepräsentation.

- Henrik Björklund, Wim Martens, Nicole Schweikardt und Thomas Schwentick schildern in ihrer Arbeit Logik und Automaten: ein echtes Dreamteam zentrale Entwicklungen der Automatentheorie im Kontext der Programmverifikation (Model-Checking) und der Datenbanktheorie.

- Martin Hoefer und Berthold Vöcking geben mit ihrem Artikel Berechnung von Nash-Gleichgewichten einen Einblick in aktuelle Fragen der algorithmischen Spieltheorie.

- Friedhelm Meyer auf der Heide und Christian Scheideler behandeln in ihrem Beitrag Algorithmische Grundlagen verteilter Speichersysteme ein Kernproblem der Theorie der Netzwerke.

- Peter Sanders stellt in seinem Artikel Algorithm Engineering einen tief greifenden Paradigmenwechsel in der Algorithmik durch die systematische Einbeziehung experimenteller Methoden vor.

- Uwe Schöning analysiert in seinem Beitrag Das SAT-Problem eines der prominentesten Grundlagenprobleme der Informatik und diskutiert u. a. die Erfolge, die sich durch den Einsatz von "SAT-Solvern" ergeben haben.

- Helmut Seidl erläutert Fortschritte in der Methodik der Programmanalyse vor dem Hintergrund einer Interaktion zwischen Theorie und Praxis.

- Martin Dietzfelbinger würdigt in einer ausführlichen Besprechung der Bücher von Ingo Wegener (1950-2008) das Werk eines herausragenden Vertreters der theoretischen Informatik. 


\section{$\{$ EDITORIAL}

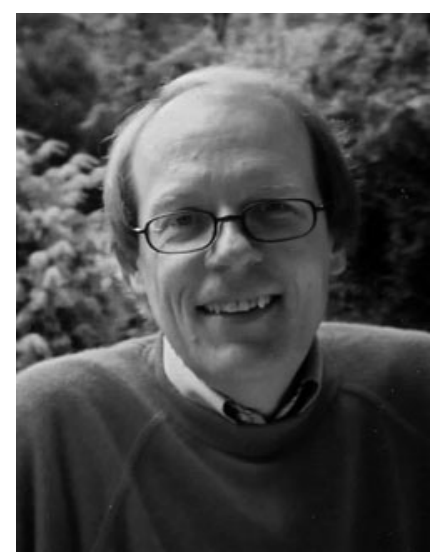

Abb. 1 Ingo Wegener 1950-2008

- In einer historischen Notiz weisen wir schließlich auf ein 100-jähriges Jubiläum hin, nämlich auf das Erscheinen einer Arbeit des norwegischen Mathematikers Axel Thue im Jahre 1910, in der viele Ideen der heutigen Theorie der Termersetzung vorgezeichnet sind.

Alle diese Beiträge sind Mosaiksteine eines umfassenderen Bildes. Es gibt eine ganze Reihe interessanter Themen, die mit gleichem Recht als profilgebend für die aktuelle Gestalt der theoretischen Informatik angesehen werden können. Über einige dieser Gebiete sind Artikel für zukünftige Hefte des Informatik-Spektrums geplant. In diesem Sinne ist das vorliegende Themenheft ein Anfang.

Zum Schluss eine Bemerkung zur Entstehung und zur Widmung des Heftes. Im Herbst 2005 fand in Schloss Dagstuhl ein Perspektiv-Workshop statt, bei dem etwa 25 Wissenschaftlerinnen und Wissenschaftler der theoretischen Informatik ihre Eindrücke und Ideen zur Entwicklung der Theoretischen Informatik zusammenführten. ${ }^{1}$ Manche Erkenntnisse und Anregungen aus diesen Diskussionen sind in die Konzeption des Themenhefts eingeflossen. Als Organisatoren des Workshops waren Ingo Wegener und der Autor dieses Vorworts bestimmt worden. Eine Woche vor dem Workshop musste sich Ingo Wegener einer Operation unterziehen. Die schwere Krankheit, der er im November 2008 schließlich erlag, hinderte ihn daran, das Projekt weiter zu begleiten. Nach längerer Zeit (im Frühjahr 2009) wurde, wieder in Dagstuhl, beim Treffen des Gl-Beirats der Universitätsprofessoren unter mehreren Teilnehmern des Workshops von 2005 beschlossen, im Informatik-Spektrum ein Themenheft zur theoretischen Informatik zu erstellen und es Ingo Wegener zu widmen. Den Herausgebern des Informatik-Spektrums und insbesondere Herrn Engesser vom Springer-Verlag sei für ihre Unterstützung bei diesem Unternehmen herzlich gedankt.

Wir, die Autorinnen und Autoren des vorliegenden Heftes und die Teilnehmerinnen und Teilnehmer des PerspektivWorkshops, widmen dieses Heft dem Andenken an Ingo Wegener als einem Kollegen, der mit einer staunenswerten Produktivität die theoretische Informatik durch zahlreiche brillante Arbeiten und Bücher bereichert hat. Von der Vielfältigkeit dieser Bereicherung zeugt insbesondere der Beitrag, den Martin Dietzfelbinger zu den Büchern Ingo Wegeners für unser Themenheft verfasst hat; dieser Text geht über eine übliche „Buchbesprechung" hinaus und bietet ein detailliertes Bild des Forschungsbereichs, den Ingo Wegener geprägt hat.

Das Titelbild des Hefts erinnert an eines der vielen Projekte, die Ingo Wegener zusammen mit seinen Studenten bearbeitet hat, nämlich den Rösselsprung auf beliebigen Schachbrettern zu analysieren (vgl. seine Arbeit Springerwege auf großen Schachbrettern, Informatik-Spektrum 15(1992), S. 172).

\footnotetext{
${ }^{1}$ Teilnehmer des Workshops waren F. Baader, W. Brauer, V. Diekert, J. Esparza, E. Grädel, M. Grohe, M. Hofmann, M. Kaufmann, M. Krause, F. Meyer auf der Heide, E.-R. Olderog, R. Reischuk, P. H. Schmitt, U. Schöning, T. Schwentick, H.P. Seidel, H. Seidl, G. Sonntag, B. Steffen, W. Thomas, D. Wagner, E. Welzl, T. Wilke und M. Wirsing.
} 
Gunter Dueck, der zusammen mit Ingo Wegener Assistent bei Rudolf Ahlswede an der Universität Bielefeld war, betrachtet in seiner Kolumne Lebensabschnittstheorien. Ihm geht es um das Entstehen und Vergehen von Theorien in der Informatik und im Leben.

Ingo Wegener war ein Wissenschaftler, der mit unbestechlichem Urteil nichts an Unklarheiten, schiefen Formulierungen oder gar Fehlern durchgehen ließ. Ingo hätte, das ist sicher, auch zu diesem Heft eine ganze Reihe konstruktiver Anmerkungen gehabt. Er konnte sie nicht mehr geben. Es ist für uns immer noch sehr schwer zu verkraften, dass er nicht mehr unter uns ist.

Mein herzlicher Dank geht an alle Kolleginnen und Kollegen, die zu diesem Heft auf ganz unterschiedliche Weise beigetragen haben.

\section{Wolfgang Thomas}

